\title{
Klimatische Zyklen im norddeutschen Vereisungsgebiet
}

\author{
Von KonRad Richter, Hannover
}

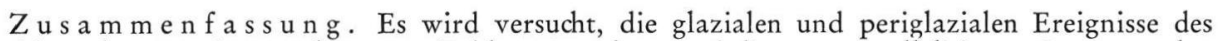
norddeutschen Vereisungsgebietes zu Zyklen zu ordnen und diese zu parallelisieren. Dazu wurden in erster Linie folgende Dokumente benutzt:

In den Lößgebieten: Bodenfließen über Dauerfrostboden, Steinsohlen, umgelagerte Lösse, Bodenbildungshorizonte, Kryoturbationen, Frostspalten, primäre Windlößsedimente; in Gebieten mit Flugsanddecken über älteren Glazialsedimenten: interstadiale Torfe, Kryoturbationen und Frostspalten; in den Gebieten mit Glazialsedimenten: Satzendmoränen, Sander, verschiedene Warwentypen, Glazialtäler, Frostspalten, Stauchmoränen, Oser und Unterschiede der Geschiebegemeinschaften von Grundmoränen.

$\mathrm{Summary}$. It is tried to divide the run of events in periglacial and in former times glaciated Regions of norther Germany to cycles and to parallellisise these. In first line these documents are used:

In the löss-areas: soilmotion above permafrost, stonelayers, removed lösses, soilbuilding, kryoturbation, icecasts, original wind-lösses; in regions with coversands and older glacialdeposits: interstadial peats, kryoturbations and icecasts; in formerly glaciated regions: normal endmoränes, outwashplanes, different types of varvic clays, glacial valleys, icekarstphänomens, pushmoränes, eskers, kames and differences in bouldercontent of boulderclays.

Seit Albrecht Penck (1909, S. 16, Fig. 1) das Schematische Bild einer glazialen Serie veröffentlichte, hat dieses Schema bis in die letzten Jahre das wissenschaftliche Schriftum so stark beeinflußt, daß Modifizierungen auf Grund neuer Erkenntnisse oft unterblieben (z. B. H. Reinhard u. G. Richter 1958, sowie H.-L. Heck 1961). Schon die aus den Jahren 1924-1926 stammenden Erkenntnisse über die besondere Art des Toteisabbaues und die Entwässerungsprobleme des letzteren in einem nach $\mathrm{S}$ ansteigenden Gelände (O. Schneider u. a. 1924 u. 1926, R. Grahmann 1937 und W. Hartnack 1926) finden nicht nur in den vorgenannten Veröffentlichungen kaum Berücksichtigung. Recht allgemein wird aus dem in Norddeutschland relativ seltenen und dann auch meist mangelhaft entwickelten Vorkommen von echten Nachschüttungssanden keine Schlußfolgerung ge zogen und ebenso wenig aus der Tatsache, daß die meisten der typischen Bändertone nach dem Hangenden zu in Vorschüttungssande bzw. Sander übergehen, während das bei anderen in Norddeutschland selteneren Typen weder an der Basis noch im Hangenden der Fall ist.

Außerdem hat in den letzten zwanzig Jahren die Erforschung periglaziärer Ablagerungen enorme Fortschritte gemacht und in Löß- sowie Flugsandgebieten klimatisch bedingte Zyklen der Sedimentation erkennen lassen. Bereits die verschiedenartige Entwicklung von Endmoränen als Satzendmoränen mit vorgelagerten Sandern und Stau- bzw. Stauchmoränen ohne zugeordnete Sander (E. LoREnTzen 1938, P. Woldstedt 1938) wurden vor dreißig Jahren als verschiedenartige klimabedingte Typen erkannt und vom Verfasser (K. Richter 1955) mit Bändertontypen und Sukzessionen von Geschiebegemeinschaften in Beziehung gesetzt.

Nunmehr ist es notwendig und erfolgversprechend, die zyklischen Entwicklungen der periglaziären Sedimentation mit jenen des glazialen Bereiches zu parallelisieren. Ein solcher Versuch kann z. Z. weder ein vollständiges noch vielleicht immer ganz r:chtiges Bild ergeben, so daß im folgenden nur einige Grundzüge genannt werden sollen.

Ein geeigneter Ansatzpunkt scheint mir das besonders eindrucksvolle Spätglazialprofil von Usseloo in den Niederlanden zu sein, in dem sowohl ein Bölling- als auch ein Alleröd- 
Torf vorkommt. Unter dem Bölling-Torf liegen Flugsande, die in einer vegetationsarmen oder -freien Zeit abgelagert wurden. Es muß also kalt und zugleich trocken gewesen sein, so daß Sande verweht werden konnten. Während der darauffolgenden Bölling-Zeit war es offenbar wärmer und feuchter, so daß Torfe entstehen konnten. Die Oberkante des Bölling-Torfes macht einen kryoturbierten Eindruck. Ein solcher kann freilich auch entstehen, wenn ein Torf über begrabenem Toteis beim Tauen desselben nach unten durchsackt, wie das K. Gripp u. a. (1964) von Meiendorf beschrieben hat. Eine solche Deutung kommt bei Usseloo also weit außerhalb der weichseleiszeitlichen Eismassen kaum infrage. Nach der Bildung der Bölling-Torfe wurde es also kälter, es blieb aber feucht, so daß Kryoturbationen möglich waren. In der Folgezeit wiederholt sich dieser Zyklus mit Flugsandablagerung, Alleröd-Torf, Kryoturbation des obersten Alleröd-Torfes und danach wieder Flugsandbildung.

Noch etwas vollständiger können klimatische Abfolgen aus Lößprofilen entwickelt werden, für die aus dem einst gletschernahen Raum nur auf die Arbeiten von $\mathrm{H}$. RoHDenburg, B. Meyer (1966) und H. Vierhuff (1967) verwiesen sei. Wir erkennen hier Abfolgen, die Fließerden, Steinsohlen, Fluglöß, Schwemmlöß, Kryoturbationen und Eiskeile enthalten, wobei letztere jeweils etwa vorausgehende Kryoturbationshorizonte durchsetzen. Letzteres finden wir auch bei Kryoturbationen von glazigenen Sedimenten. Ein Beispiel dafür bildet B. Heinemann (1964) ab, wo in Spahn (Hümmling) eine kryoturbierte Sandtasche in Geschiebelehm von einer Frostspalte durchsetzt wird. Die Kryoturbation erfolgte also in feuchter Kaltzeit und die Frostspaltenbildung danach in einer noch kälteren und trockeneren Periode, in der das Eis des gefrorenen Bodens sein Volumen wieder verringerte. Beobachtungen über eine Frostspalte, die offenbar eine wieder gefrorene flaserige Fließerde durchsetzt, wird R. MARCZINSKI in Kürze in seiner Dissertation veröffentlichen. Die meist weichseleiszeitlich gebildete und im nördlichen Niedersachsen weit verbreitete Steinsohle dürfte in einer feucht-kalten Zeit gleichzeitig mit Fließerdebildungen erfolgt sein. Bei Lorup im Hümmling war z. B. 1946 sehr schön zu sehen, wie eine dünne Geschiebelehmdecke sich bei schwacher Hangneigung in Kryoturbationskissen auflöste, die hangabwärts kleiner wurden und in eine Steinsohle übergingen. Diese wurde danach in einer trocken-kalten Zeit teilweise windpoliert, so daß zahlreiche Windkanter entstanden. Es gibt indes auch kryoturbierte Steinsohlen, in denen Windkanter mit verbrodelt sind. Diese entstammen offenbar einer älteren trocken-kalten Phase.

Zyklische Phänomene glazigener Sedimente des norddeutschen Vereisungsgebietes wurden vom Verfasser bereits früher (z. B. 1937 und 1957) dargestellt. In letzterer Arbeit sind insbesondere Beziehungen zwischen verschiedenartigen Endmoränentypen, Bändertonen und westlich wie östlich beheimateten nordischen Geschiebegemeinschaften aufgezeigt. Am deutlichsten klimatisch auswertbar sind dabei die verschiedenen Bändertontypen in Norddeutschland. Außer dem in Schweden vorwiegend vorkommenden Normaltypus, bei dem Sommer- und Winterlagen nicht allzu große Mächtigkeitsunterschiede haben, sind in Norddeutschland Typen viel häufiger, bei denen die Sommerlagen sehr viel dicker sind als die fetteren der Winter und in einem Typus 30-40, seltener bis 80 Tageswarven erkennen läßt, während der andere oft über 100 Tageswarven zeigt. Beide enthalten im mittleren Teil der jeweiligen Tageswarven Kriechspuren, z. B. von Käfern und Wasserrasseln. Die Bildungszeit war also nicht absolut lebensfeindlich, was mindestens zum Teil auch auf Oser zutrifft. Anläßlich einer Tagung der Deutschen Geologischen Gesellschaft in Lübeck 1933, führte W. WolfF z. B. den Swinkuhler Os in Oolstein vor, in dem sich eine Lage teilweise zweiklappiger Unionen befand. Auch H.-L. Heck (1961, S. 388) erwähnt eine Mitteilung von A. Ludwig (1960), wonach sich in einem vom Eise überfahrenen Tonschluff westlich Sternberg i. Mecklenburg eine ganze Anzahl zweiklappiger Unionen befand. Die Klimabedingungen werden von H.-L. Heck infolgedessen als „interstadial“ beurteilt. 
Außerdem kommen zumindest in der Toteislandschaft zwischen dem mittelpommerschen Hauptstaumoränenzug und der Satzendmoräne des Pommerschen Stadiums noch weitere Bändertone vor, in denen die Unterscheidung von Sommer-, Winter- und Tageslagen äußerst schwierig bzw. unmöglich ist. Aus Ostpommern erwähnt M. VIERKE (1937) einen solchen von Damsdorf bei Bütow. Ich selbst fand diesen Typus nicht weit von den im Pyritzer Weizacker sonst überwiegend verbreiteten Bändertonen, die klare Sommerund Winterlagen enthalten, außerdem bei Fürstensee. Auch unweit Neukalen in Mecklenburg, war der unklare Typus 1936 aufgeschlossen. Wir haben also in Norddeutschland in jeweils spezifischer glazigener Position mindestens 4 Typen von Bändertonen, die offenbar unter verschiedenartigen klimatischen Bedingungen gebildet wurden.

Von den morphologischen Formen des norddeutschen Vereisungsgebietes weisen auch einige auf unterschiedliche Klimabedingungen hin. Bei der Ablagerung von Satzendmoränen herrschte offenbar relativ feucht-milde Witterung mit starker Schmelzwasserproduktion, Aufschüttung von Sandern und breiten Spalten - und Tunneltälern im Rückland. Dagegen war während der Bildung von Staumoränen der Boden vor dem vorrückenden und auch unter stationärem Eis gefroren, so daß kaum ein großer Festigkeitsunterschied zwischen der an dirt-bands reichen Basis der Gletscher und dem gefrorenen Untergrund bestand. So kam es beim Vorrücken der Gletscher zum Mitreißen von Detraktionsschollen des Untergrundes und zur Bildung von Stau- bzw. Stauchmoränen. $\mathrm{Da}$ ein solches Vorrücken der Gletscher unter besonders kalten und trockenen, also ernährungsfeindlichen Bedingungen oft nicht ausreichte, die Inlandeismassen bis zum äußersten Rand zu aktivieren, bildeten sich Stauchmoränen an der Aktivierungsgrenze gegen ein Toteisgebiet. Dafür seien zwei Beispiele gebracht: Aus dem Jungmoränengebiet sei der Mittelpommersche Hauptstaumoränenzug genannt, der in Vorpommern am Südrand des Pommerschen Urstromtales liegt. In diesem flossen die verhältnismäßig geringen Schmelzwässer dieser mehr trockenen kalten Zeit anschließend nach Westen. Ein Beispiel aus dem Altmoränengebiet sind die Dammer- und Fürstenauer Berge des Rehburger Stadiums. An der Grenze zwischen aktiviertem und totem Eisgürtel kam es natürlich gelegentlich zu lokalen Überfahrungen, die von manchen Autoren (G. KelleR 1953) sicher überschätzt wurden. Der Mittelpommersche Hauptstaumoränenzug ist z. B. in Vorpommern nicht, bei Stettin wenig, in Ostpommern (Glockenberge) dagegen stärker überfahren. Die Reaktivierung des Oder-Gletschers war in der Westflanke offenbar geringer als in der Ostflanke. Entsprechend ist das sogenannte Pommersche Urstromtal im Zuge der Schwächezone zwischen schwach aktiviertem Toteisgürtel und reaktiviertem Eis in Hinterpommern als System von nacheinander entleerten Staubecken (z. B. W. HARTNAck 1926), in Vorpommern dagegen als Stromtal mit stellenweise umfangreichen Geröllbänken entwickelt. Auch das Land nördlich des Pommerschen Urstromtales wurde in der Zeit der vorgenannten Staubecken wieder Toteisgebiet. Besonders in Vorpommern hatten sich gleichzeitig mit der Satzendmoränenbildung des Pommerschen Stadiums breite Radialtäler entwickelt. Sie sind oft so breit, daß sie in Anbetracht der wahrscheinlich nicht sehr großen Eismächtigkeit zumeist nicht als Tunnel-, sondern als Spaltentäler anzusprechen sind, wie ja auch der grönländische heutige Eisrand streckenweise breite und lange offene Spalten zeigt (s. Abb. 247 in Viete-Wagenbreth-Hunger-Reichert 1960). In der darauffolgenden Toteisphase konnte bei dem kälteren Klima die geringere Schmelzwasserproduktion nicht mehr die ganze Talbreite offen halten. Infolge gewisser Plastizität des Eises und in Anbetracht der Dicke der Eismasse hatten die Spalten eine Tendenz sich zu schließen bzw. zu verengen. Als es wieder feuchter und - wie gelegentliche Lebensspuren (Unionen) zeigen auch wärmer wurde, bildeten sich in den Restspalten und -tunneln Oser (K. Richter, 1937).

Daß die Dammer- und Fürstenauer-Berge als Stauchmoräne an der Grenze von reaktiviertem gegen Toteis entstanden, wurde schon 1961 von mir vor allem mit dem Feh- 
len einer Grundmoräne auf dem aufgeschuppten Gebiet begründet. Auch die Oberflächenformen des südlichen Vorlandes scheinen mir dafür zu sprechen. Während die Stauchmoränen selbst aus einer großen Anzahl eisrandparalleler Rücken bestehen, die im Kern eine Detraktionsscholle von meist tertiären Tonen enthalten (Bundesautobahnaufschlüsse 1967), verlaufen die Höhenrücken im südlichen Vorland in der Bewegungsrichtung des Eises. Sowohl vor den Fürstenauer- wie vor den Dammer-Bergen ist je ein solcher etwas kuppiger Rücken besonders gut entwickelt. Vor den Fürstenauer-Bergen ist das der schlecht aufgeschlossene Dingerberg und vor den Dammer Bergen in und südlich Sierhausen ein morphologisch sehr gleichartiger Rücken, der im Frühjahr 1968 leidlich aufgeschlossen war. Noch kurz nördlich des Dingerberges waren schöne eisrandparallele Stauchungen zu sehen. Die Aufschlüsse im Rücken bei Sierhausen zeigten keinerlei Stauchungen, sondern horizontalschichtige Sande und Kiese mit $1 \mathrm{~cm}$ dicken Schlufflagen. Im südlichen Abschnitt kamen randnah in den Sanden kleine durch Toteis verursachte Verwerfungen vor. Man könnte in beiden Fällen an in Eisspalten gebildete Oser denken. Die Rücken erinnern aber mehr an die Kames, welche J. Elbert (1904) besonders vom Südrand des Mittelpommerschen Hauptstaumoränenzuges aus Vorpommern und Mecklenburg beschrieb. Die morphologische Ausbildung und die morphologische Gesamtsituation insbesondere der Weltziner Kames-Gruppen und der Rücken südlich der Dammer-Fürstenauer-Berge sind sehr ähnlich. Noch deutlicher als bei Weltzin ist der Kame bei Kierhausen keine Spaltenfüllung durch ein viele Kilometer langfließendes Gewässer, sondern die lokale Füllung einer Kurzspalte, die im Zuge der Stauchmoränenaufschuppung im südlich davor gelegenen Toteisgebiet aufriß. Eine Grundmoränenbedeckung fehlt.

Aufgrund der vorstehend angeführten klimatischen Deutungen könnte man versuchen, die Ereignisse der periglaziären und glazialen Ablagerungsräume zu parallelisieren und tabellarisch darzustellen. Dabei würden sich noch einige Unklarheiten zeigen, zumal Sedimentationszyklen in der Erdgeschichte sehr oft nicht vollständig entwickelt sind und vielfach Teilzyklen einander folgen.

Es sei daher zusammenfassend auf einige wahrscheinliche Parallelisierungsmöglichkeiten hingewiesen, wobei die gesamten Zyklenfolgen in ihren Grundzügen wahrscheinlich noch nicht voll erfaßt sind:

1. Fließerden, Schwemmlösse und Bodenhorizonte der Lößgebiete entsprechen nach der klimatischen Genese mehr oder weniger den interstadialen Torfen, z. B. des Bölling und Alleröd, z. T. auch der Steinsohlenbildung in den Flugsandgebieten sowie den Satzendmoränen, Sandern, breiten Spaltentälern und Bändertonen mit jeweils 30 bis 80 Tageswarmen und z. T. auch wohl Osern des vergletschert gewesenen Gebietes. Geschiebekundlich sind die letzteren Gebiete westskandinavisch geprägt. Das Eis des westskandinavischen Eiszentrums wurde also durch viel Feuchtigkeit gut ernährt und konnte daher in den norddeutschen Raum vorstoßen.

2. Das Klima bleibt danach noch feucht, wenn auch wohl trockener als vorher. Es wird aber sicher kälter, so daß sich in den periglaziären Gebieten sowohl Kryoturbationen in älteren glazialen Sedimenten als auch in den Torfen der Flugsandgebiete und in den Lössen entwickeln konnten. Die vergletscherten Gebiete entwickelten Toteismassen und Eiskarstphänomene, da der Nachschub aus dem westskandinavischen Eiszentrum nachließ. Die Spaltentäler wurden infolge der Eisplastizität und des geringen Schmelzwasseranfalles enger. Die Bändertonbildung beschränkte sich auf kleinere Gebiete, war aber besonders reich an Tageswarwen. Das erscheint widersprüchlich. Vielleicht ist es aber durch geringere Bewölkung zu erklären, so daß zwar häufig ein mittägliches Schmelzen eintrat, die Schmelzwässer aber wegen der bald wieder einsetzenden täglichen Abkühlung nicht weit transportiert wurden. Geschiebekundlich liegen die Heimatgebiete jetzt östlicher als unter Ziffer 1. 
3. Das Klima wurde allmählich trockener und kälter. In den Periglazialgebieten bildeten sich Eisspalten, welche oft die wiedergefrorenen Kryoturbationszonen auch in den Lössen durchsetzten. In den vergletscherten Gebieten erlahmte der Nachschub des Westeises so stark, daß vom ostskandinavischen Eiszentrum her die Eismassen der Ostseedepression folgen und somit in Norddeutschland vorstoßen konnten. Es gelang diesem Eis aber nicht mehr, die Toteismassen in Norddeutschland bis zum derzeitigen äußersten Rand zu aktivieren. Der Boden war wegen der hohen Kälte unter dem schon durch Ablation dünner gewordenen Eis gefroren. Es kam unter dem aktiven Eis zum Losreißen von großen Detraktionsschollen und an der Grenze zum Toteissaum zu Stau- bzw. Stauchmoränenaufschuppungen. Die Schmelzwasserentwicklung war bei der großen Kälte sehr gering, so daß Sanderbildungen fehlen. Immerhin war, wie schon unter Ziffer 2 angenommen, der Bewölkungsschirm wahrscheinlich noch geringer und lokale mittägliche Schmelzwässer bildeten sehr selten räumlich begrenzte Bändertone, in denen Winter-, Sommer- und Tageslagen kaum zu identifizieren sind. Ob die Bildung der Oser in den verengten Spaltentälern schon jetzt einsetzte oder, worauf Unionenfunde hindeuten, erst im Ubbergang $\mathrm{zu}$ der normalerweise wieder folgenden Phase 1, wage ich noch nicht zu entscheiden. Die Geschiebeführung des „Ostseegletschers" wurde nunmehr überwiegend vom ostskandinavischen Eiszentrum geprägt und enthält sehr viele Gesteine von den Åland-Inseln und aus Finnland. Ein Teil der westskandinavischen Geschiebe dieses Eisstromes ist sicher als Reprise zu erklären, da Exarationen und in noch stärkerem Maße Detraktionen zu dieser Zeit besonders intensiv waren.

Ich bin mir im klaren darüber, daß die vorstehenden Parallelisierungsversuche weder vollständig, noch voll befriedigend sind. Die grundsätzlichen Ges:chtspunkte dürften aber eine weitere Durcharbeitung anregen.

\section{Literatur}

Elbert, J.: Die Entwicklung des Bodenreliefs von Vorpommern und Rügen, sowie den angrenzenden Gebieten der Uckermark und Mecklenburgs während der letzten diluvialen Vereisung. - Jahresber. d. Geogr. Ges. zu Greifswald, VIII, 141-243, Greifswald 1904.

Grahmann, R.: Form und Entwässerung des nordeuropäischen Inlandeises. Mitt. d. Ges. f. Erdk. zu Leipzig 54, 48-70, Leipzig 1937.

GrIPP, K.: Erdgeschichte von Schleswig-Holstein. Neumünster 1964.

Hartnack, W.: Die Küste Hinterpommerns. II. Beiheft z. Jahrb. d. Geogr. Ges. 43-44, Greifswald 1926.

Heck, H.-L.: Glaziale und glaziäre Zyklen. Geologie 10, 378-395, Berlin 1961.

Heinemann, B.: Die Böden der Drenthe-Grundmoräne im Hümmling. Diss. Hannover 1964.

Keller, G.: Die Beziehung des Rehburger Stadiums südlich Ankum (Kr. Bersenbrück) zur saaleeiszeitlichen Grundmoräne. Eiszeitalt. u. Gegenw. 3, 58-64, OOhringen 1953.

LoREntzen, E.: Die Oberflächenformen der Landschaft Angeln und ihre Abhängigkeit vom Klimawechsel während der jüngsten Vereisung. Schrift. d. Nat.wiss. Ver. für Schleswig-Holstein 22, Kiel 1938.

Penck, A. \& BrüCKner, E.: Die Alpen im Eiszeitalter. 1, Leipzig 1909.

Reinhard, H. \& Richter, G.: Zur Genese der Gletscherzungenbecken Norddeutschlands. Z. Geomorph. 2, 55-75, Berlin 1958.

Richter, K.: Gefüge und Zusammensetzung des norddeutschen Jungmoränengebietes. Abh. Geol.Pal. Inst. Greifswald 1-63, Greifswald 1933. - - Die Eiszeit in Norddeutschland Verl. Bornträger, Berlin 1937. - - Klimatische Verschiedenartigkeit glazialer Vorstoßphasen in Norddeutschland. Act. IV, Congr. intern. Quaternaire, Roma 1955. - - AufpressungsartigeGletscherbruchrücken südlich Cloppenburg in Oldenburg. Z. deutsch. geol. Ges. 112, 369-377, Hannover 1961.

Rohdenburg, H. \& Meyer, B.: Zur Feinstratigraphie und Paläopedologie des Jungpleistozäns nach Untersuchungen an süd-niedersächsischen und nordhessischen Lößprofilen. Mitt. d. Deutsch. Bodenkundl. Ges. 5, 1-137, Göttingen 1966. 
RoHDEnBurg, H.: Eiskeilhorizonte in südniedersächsischen und nordhessischen Lößprofilen. Mitt. d. Deutsch. Bodenk. Ges. 5, 137-170, Göttingen 1966.

Schneider, O.: Was uns Pommerns Berge erzählen. Unser Pommerland 9, Stettin 1924. - - Uberblick über den geologischen Bau Pommerns. Das Pommersche Heimatbuch, 1-70, Berlin 1926.

VIERHUfF, H.: Untersuchungen zur Stratigraphie und Genese der Sandlößvorkommen in Niedersachsen. Mitt. Geol. Inst. T.H. Hannover, Heft 5, Hannover 1967.

VIERKE, M.: Die ostpommerschen Bändertone als Zeitmarken und Klimazeugen. Diss. Greifswald 1937.

G. Viete - O. Wagenbreth - R. Hunger-H. Reichert: Geologie 1, Bergakademie Freiberg, Fernstudium, Freiberg i. Sa. 1960.

Woldstedt, P.: Über Vorstoß und Rückzugsfronten des Inlandeises in Norddeutschland. Geol. Rundsch. 29, Stuttgart 1938.

Manuskr. eingeg. 2. 9. 1968.

Anschrift des Verf.: Prof. Dr. Konrad Richter, Hannover, Welfengarten 1, Institut für Geologie und Paläontologie der Technischen Universität Hannover. 